\title{
High-Temperature Superconducting Cable Fault Location Method Based on Improved Time-Frequency Domain Reflection Method and EEMD Noise Reduction
}

\author{
Bo Yang, ${ }^{1}$ Jun Tang, ${ }^{1}$ Chen Yang, ${ }^{1}$ Xiaofeng Dong, ${ }^{1}$ Kun Huang, ${ }^{2,3}$ and Changsen Feng $\mathbb{D}^{4}$ \\ ${ }^{1}$ State Grid Suzhou Power Supply Company, Suzhou 215004, China \\ ${ }^{2}$ Nari Technology Co., Ltd., Nanjing 211100, China \\ ${ }^{3}$ Nanjing University of Posts and Telecommunications, Nanjing 210023, China \\ ${ }^{4}$ College of Information Engineering, Zhejiang University of Technology, Hangzhou, 310023, China
}

Correspondence should be addressed to Changsen Feng; fcs@zjut.edu.cn

Received 20 October 2021; Accepted 7 November 2021; Published 2 December 2021

Academic Editor: Jinyan Song

Copyright $\odot 2021$ Bo Yang et al. This is an open access article distributed under the Creative Commons Attribution License, which permits unrestricted use, distribution, and reproduction in any medium, provided the original work is properly cited.

\begin{abstract}
Aiming at the operation and maintenance requirements of the fault location of high-temperature superconducting cables, a fault location method of high-temperature superconducting cables based on the improved time-frequency domain reflection method and EEMD noise reduction is proposed. Considering the cross-term interference problem in the traditional time-frequency domain reflection method, this paper introduces the affine transformation to project the time-frequency distribution of the selfterm and the cross term and further highlights the characteristic differences between the two through coordinate transformation, and the particle swarm algorithm is employed to solve the optimal stagger angle of the affine transformation. The unscented particle filter is adopted to separate the cross term, and EEMD noise reduction is introduced to solve the signal noise problem. Finally, two software programs, PSCAD and MATLAB, are employed for joint simulation to build a model of high-temperature superconducting cable. The simulation example shows that the proposed method in this paper can eliminate the cross-term interference of the traditional time-frequency domain reflection method, effectively locate the fault of the high-temperature superconducting cable, and improve the positioning accuracy.
\end{abstract}

\section{Introduction}

In recent years, with the continuous development of social economy, conventional urban cable transmission capacity is small, covers a large area, and has high line loss, so the shortcomings such as the difficulty of grid expansion are increasingly prominent, which restricts the development of smart grid [1-3]. Compared with conventional cables, hightemperature superconducting cables take advantage of the non-resistance and high current density properties of superconducting materials in the superconducting state and have significant advantages such as high capacity, low line loss, light weight, and environmental friendliness, providing an efficient and environmentally friendly way to transmit over long distances for smart grids [4-7]. Currently, several countries around the world are planning to advance the large-scale commercial application of high-temperature superconducting cables in an orderly manner $[8,9]$. Accurately locating high-temperature superconducting cable faults and exploring practical, effective, and convenient fault detection methods can help to quickly remove faults and repair them to ensure the safe and stable operation of the power systems.

At present, there have been many studies on cable fault location, mainly focusing on the low-voltage pulse reflection method based on traveling wave theory [10], pulse current method [11], pulse voltage method [12], wavelet analysis method [13], and so on, while there are very few results dedicated to the study of high-temperature superconducting cable fault location. Based on distributed optical fiber temperature measurement technology, Huang et al. [14] proposed a local loss of superconducting cable detection and 
protection method, which can effectively respond to local loss of superfault, alarming, and tripping according to the size and temperature of the heat generating area. Wang et al. [15] conducted a high-temperature superconducting cable fault location study based on time-frequency domain reflectometry (TFDR) and introduced the pseudo-WignerVille distribution (PWVD) to analyze the time-frequency of the signal. A real $35 \mathrm{kV}$ high-temperature superconducting cable is analyzed for the variation pattern of waveforms at different temperatures, different fault types, and different signal parameters. Although the time-frequency domain analysis method is more advantageous than the single-domain analysis method when the reflected signal is weak and the signal attenuation is large, the introduction of the PWVD will generate cross-term interference when multiple signals are superimposed, which will seriously affect the analysis results. In [16-20], generalized regression neural network, heterogeneous cuckoo algorithm, analysis of the time domain energy of the reflected signal, analysis of the Euclidean distance and tangent distance of the incoming reflection time-frequency distribution, and affine transformation were proposed for this problem, which effectively improved the traditional time-frequency domain analysis method to some extent.

Signals are often noisy during transmission, and the accuracy of fault location can be improved by using suitable noise reduction methods. In literature [21], empirical mode decomposition (EMD) was used to reduce noise in the echo signal of full-waveform laser altimetry system for complex scenes with multiple height layers, and a good noise reduction effect was achieved. However, the EMD suffers from mode aliasing, which limits the noise reduction effect. To overcome this shortage, this paper introduces the ensemble empirical mode decomposition (EEMD) [22, 23] method, which introduces Gaussian white noise to maximize the retention of the real signal.

This paper proposes a fault location method for hightemperature superconducting cables based on the improved time-frequency domain reflection method and EEMD noise reduction. Firstly, the affine transformation is introduced to solve the cross-term interference problem of time-frequency domain reflection method, and the particle swarm algorithm is applied to solve the problem of optimal stagger angle accuracy, and the cross-term separation is achieved by the unscented particle filter algorithm. Secondly, the signal is denoised by EEMD to make the positioning results more accurate. Finally, the simulation environment is built to verify the validity and accuracy of the model in this paper.

\section{Improved Time-Frequency Domain Reflection Method}

2.1. Traditional TFDR. As a cable fault non-destructive detection method, TFDR sends an incident signal to the cable and uses a digital oscilloscope to pick up the folded reflection signal generated by the incident signal at the point of impedance discontinuity, so as to locate the high-temperature superconducting cable fault location, and the schematic diagram is described in Figure 1.
The incident signal is usually a linearly modulated signal with Gaussian envelope that provides localized information in the time and frequency domains, and its mathematical expression is

$$
s(t)=\left(\frac{\alpha}{\pi}\right)^{1 / 4} e^{-\alpha\left(t-t_{0}\right)^{2} / 2+\mu \beta\left(t-t_{0}\right)^{2} / 2+\mu \omega_{0}\left(t-t_{0}\right)},
$$

where $\alpha$ is the duration of the signal, $t_{0}$ is the time center of the signal, $\mu$ is the bandwidth of the signal, and $\omega_{0}$ is the center frequency of the signal. The signal pulse width becomes narrower when $\alpha$ increases, and the signal bandwidth becomes wider when $\beta$ increases.

The incident signal produces folded reflection at the point of impedance discontinuity, and the reflected signal is correlated with the incident signal in the time-frequency domain. TFDR uses the Wigner-Ville distribution (WVD) to analyze the signal in the time-frequency domain, that is,

$$
W(t, \omega)=\int_{-\infty}^{+\infty} s\left(t+\frac{\tau}{2}\right) s^{*}\left(t-\frac{\tau}{2}\right) e^{-b \omega \tau} \mathrm{d} \tau,
$$

where $s(t)$ is the incident signal, $\omega$ denotes the frequency, and $\tau$ and $b$ are the signal parameters.

TFDR calculates the time-frequency correlation function of the incident and reflected signals.

$$
C_{s r}(t)=\frac{2 \pi}{E_{s} E_{r}(t)} \int_{t^{\prime}=t-T_{s}}^{t^{\prime}=t+T_{s}} \int W_{r}\left(t^{\prime}, \omega\right) W_{s}\left(t^{\prime}-t, \omega\right) \mathrm{d} \omega \mathrm{d} t
$$

where $E s$ is the time-frequency domain energy of the incident signal, Er $(t)$ is the time-frequency domain energy of the reflected signal, and $\mathrm{Wr}(t, \omega)$ and $\mathrm{Ws}(t, \omega)$ are the WVDs of the reflected signal and the incident signal. The time-frequency correlation function $C_{s r}(t)$ will present a local peak when $W_{r}$ and $W_{s}$ match, and the peak position can be used to detect the fault location. Suppose that the time difference between the incident signal reflected at the head point of the HTS cable and the local peak reflected at the fault point is $\Delta t$; then, the distance between the fault point and the head point of the cable can be calculated as

$$
l=\frac{(v \times \Delta t)}{2}
$$

where $v$ is the propagation speed of the signal.

Due to the attenuation of the incident signal as it propagates in the high-temperature superconducting cable, both the time center and frequency center of the signal are shifted, requiring a time compensation for the time difference $\Delta t$.

$$
\Delta t^{\prime}=\Delta t+\frac{\delta_{\omega}}{\theta}
$$

where $\delta_{\omega}$ is the frequency offset and $\theta$ is the waveform parameter.

2.2. Affine Transformation. Conventional TFDR faces the cross-term interference problem, thus causing misclassification. References $[15,17]$ use the windowing strategy to 


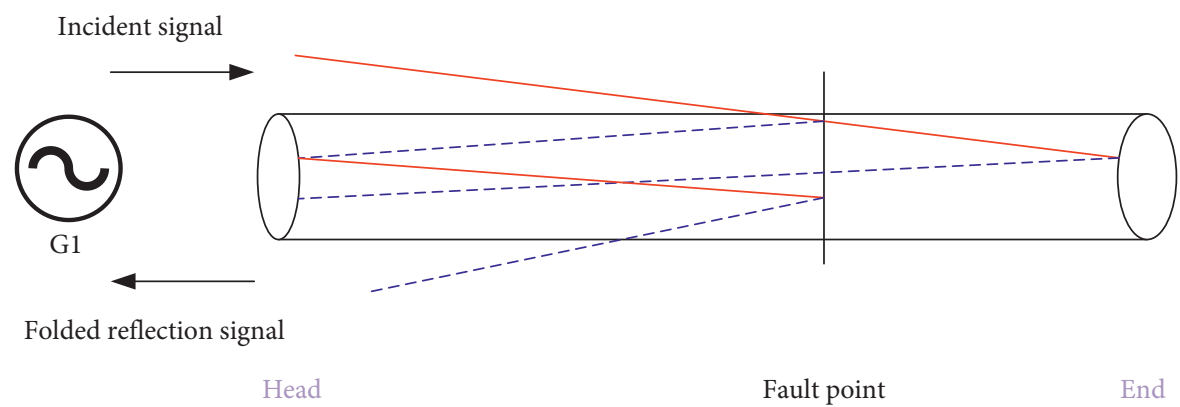

FIgURE 1: TFDR schematic.

suppress the cross terms, but it is easy to interfere with the self-term and reduce the localization resolution. Studies have shown that the lateral characteristics of the self-term and cross term differ significantly, and the self-term energy distribution is concentrated and smooth, while the crossterm distribution exhibits positive and negative oscillations [20]. Therefore, the time-frequency distributions of the selfterm and cross term can be considered for projection, and the differences in the characteristics of the two can be further highlighted by means of coordinate transformation, and the cross term can be separated by means of filtering.

The affine transformation is a two-dimensional planar transformation method widely used in the field of image processing with homogeneity and union, and the features of the transformed figure still retain their original characteristics $[24,25]$. The affine transformation includes rotation, stretching, stagger, translation, and other change means, and a straight line is still a straight line after affine transformation, and a parallel line is still a parallel line after affine transformation. The affine transformation is employed to project the time-frequency distribution of TFDR to be parallel to the coordinate axis, which can be analyzed in single time domain or single frequency domain.

The key of affine transformation is to obtain the affine transformation matrix, and the result matrix after affine transformation can be obtained by the product of this matrix and the time-frequency distribution matrix. The solution process is usually based on the Fourier transform to find the matching expression of the spectral integral curve in logarithmic polar coordinates and then to find the extreme value point of the curve or to directly fit the curve after the transformation non-linearly. In the literature [20], the solution process is simplified by using the affine transformation with a cross-axis tangent based on the characteristics of the self-term and cross term obtained by TFDR for hightemperature superconducting cables. The affine transformation matrix $M$ of the cross-axis stagger can be expressed as

$$
M=\left[\begin{array}{ccc}
1 & 0 & 0 \\
-\frac{1}{k} & 0 & 0 \\
0 & 0 & 1
\end{array}\right] .
$$

The optimal stagger angle $q$ can be formulated as

$$
q=\arg \max \left(\max \left(\operatorname{sum}\left(\operatorname{col}\left(\mathbf{M}_{v}\right)\right)\right)\right),
$$

where argmax () denotes the value corresponding to the maximum value when taken, col () represents each column of the matrix, and $\mathbf{M} v$ denotes the transformed time-frequency matrix. The selection of $q$ value directly affects the accuracy of affine transformation and fault location.

2.3. Particle Swarm Algorithm. Particle swarm algorithm is a common artificial intelligence optimization algorithm, which has the advantages of simplicity, easy implementation, and fast convergence compared to the genetic algorithms and simulated annealing methods [26, 27]. Its iterative process can be described as follows:

$$
\left\{\begin{array}{l}
\operatorname{pv}^{m+1}(i)=u \cdot \mathrm{pv}^{m}(i)+d_{1} r_{1}\left(\mathrm{pBest}^{m}-\mathrm{px}^{m}(i)\right)+d_{2} r_{2}\left(g \mathrm{Best}^{m}-\mathrm{px}^{m}(i)\right), \\
\mathrm{px}^{m+1}(i)=\mathrm{px}^{m}(i)+\mathrm{pv}^{m+1}(i),
\end{array}\right.
$$

where $\mathrm{pv}^{m}(i)$ and $\mathrm{px}^{m}(i)$ are the velocity and the position of particle $i$ at the $m_{\text {th }}$ generation, respectively, $u$ is the inertia weight factor, $d_{1}$ and $d_{2}$ are the local and global learning factors, respectively, $r_{1}$ and $r_{2}$ are random numbers between 0 and 1 , pBest $^{m}$ denotes the historical optimal position of particle $i$ at each iteration, and $g$ Best $^{m}$ represents the global optimal position of the whole particle swarm at each iteration.
In this paper, the particle swarm algorithm is used to solve the optimal stagger angle, and the affine transformation matrix can be obtained. In order to maintain the diversity of the population, this paper draws on the idea of genetic algorithm and introduces the variation operator into the particle swarm algorithm, where the particle positions may be mutated at each iteration, and its expression is as follows: 


$$
\left\{\begin{array}{l}
a^{m}=1-\lambda^{(1-m / N)^{2}}, R_{1}>0.7 \\
\operatorname{px}^{m+1}(i)^{\prime}=\mathrm{px}^{m+1}(i)+a^{m} \cdot \mathrm{px}^{m+1}(i) \cdot R_{1}, R_{2}>0.5 \\
\mathrm{px}^{m+1}(i)^{\prime}=\mathrm{px}^{m+1}(i)-a^{m} \cdot \mathrm{px}^{m+1}(i) \cdot R_{1}, R_{2} \leq 0.5
\end{array}\right.
$$

where $a^{m}$ is the mutation operator after the $m_{\mathrm{th}}$ iteration, $\lambda$ is the mutation parameter in the range of $[0,1], N$ is the preset maximum number of iterations, $R_{1}$ and $R_{2}$ are both random numbers in the range of $[0,1]$, and $\mathrm{px}^{m+1}(i)^{\prime}$ denotes the new particle position after the $m_{\text {th }}$ iteration after mutation. At the beginning of the iteration, $a^{m}$ takes a larger value to strengthen the global search ability of the particle, while at the end of the iteration, $a^{m}$ takes a smaller value to strengthen the local search ability of the particle.

2.4. Unscented Particle Filter. After affine transformation, the self-term is distributed in the low-frequency part and the cross term is distributed in the high-frequency part, so the cross term can be eliminated by low-pass filtering. This method does not affect the self-term resolution, which is theoretically more advantageous than the window addition method.

The unscented particle filter method is based on Bayesian estimation theory and is very suitable for non-Gaussian nonlinear systems with high estimation accuracy and fast computational efficiency and is widely used in modern signal processing $[28,29]$. Its filtering steps are as follows:

(1) Initialization is performed using the following expression:

$$
\left\{\begin{array}{l}
\mathbf{x}_{0}^{i}=E\left[\mathbf{x}_{0}^{i}\right], k=0, \\
\mathbf{P}_{0}^{i}=E\left[\left(\mathbf{x}_{0}^{i}-\bar{x}_{0}^{i}\right)\left(\mathbf{x}_{0}^{i}-\bar{x}_{0}^{i}\right)^{T}\right], k=0, \\
\bar{x}_{0}^{i a}=E\left[\bar{x}_{0}^{i a}\right], k=0,
\end{array}\right.
$$

where $x_{0}{ }^{i}$ denotes the initial state variable at the number of iterations $k=0, \boldsymbol{P}_{0}{ }^{i}$ is the covariance matrix, and $\bar{x}_{0}^{i a}$ represents the original particle set. When $k$ is greater than 0 , let $k=k+1$ iterations to get a new round of particle set.

(2) Calculate the sigma point set, conduct one-step prediction, and calculate the covariance matrix:

$\mathbf{x}_{k \mid k-1}^{i a}=f\left(\mathbf{x}_{k-1}^{i x}, i_{k-1}\right)$,

$\bar{x}_{k \mid k-1}^{i}=\sum_{j=0}^{2 n_{a}} W_{j}^{m} \mathbf{x}_{j, k \mid k-1}^{i x}$,

$\mathbf{P}_{k \mid k-1}^{i}=\sum_{j=0}^{2 n_{a}} W_{j}^{c}\left[\mathbf{x}_{j, k \mid k-1}^{i x}-\bar{x}_{k \mid k-1}^{i}\right]\left[\mathbf{x}_{j, k \mid k-1}^{i x}-\bar{x}_{k \mid k-1}^{i}\right]^{T}$.

(3) Calculate the observed predictions:

$$
\begin{aligned}
& \mathbf{z}_{k \mid k-1}^{i}=F\left(\mathbf{x}_{k \mid k-1}^{i x}, i_{k-1}\right), \\
& \bar{z}_{k \mid k-1}^{i}=\sum_{j=0}^{2 n_{a}} W_{j}^{c} \mathbf{z}_{j, k \mid k+1}^{j} .
\end{aligned}
$$

(4) Calculate the system covariance matrix according to the weighted values:

$$
\begin{aligned}
& \mathbf{P}_{z_{k} z_{k}}=\sum_{j=0}^{2 n_{a}} W_{j}^{c}\left[\mathbf{z}_{j, k \mid k-1}^{i}-\bar{z}_{k \mid k-1}^{i}\right]\left[\mathbf{z}_{j, k \mid k-1}^{i}-\bar{z}_{k \mid k-1}^{i}\right]^{T}, \\
& \mathbf{P}_{x_{k} z_{k}}=\sum_{j=0}^{2 n_{a}} W_{j}^{c}\left[\mathbf{x}_{j, k \mid k-1}^{i}-\bar{x}_{k \mid k-1}^{i}\right]\left[\mathbf{x}_{j, k \mid k-1}^{i}-\bar{x}_{k \mid k-1}^{i}\right]^{T} .
\end{aligned}
$$

(5) Calculate the Kalman gain and update the system state and covariance matrices:

$$
\begin{aligned}
\mathbf{K}_{k} & =\mathbf{P}_{x_{k} z_{k}} \mathbf{P}_{z_{k} z_{k}}^{-1}, \\
\bar{x}_{k}^{i} & =\bar{x}_{k \mid k-1}^{i}+\mathbf{K}_{k}\left(\mathbf{z}_{k}-\bar{z}_{k \mid k-1}^{i}\right), \\
\widehat{P}_{k}^{i} & =\mathbf{P}_{k \mid k-1}^{i}-\mathbf{K}_{k} \mathbf{P}_{z_{k} z_{k}} \mathbf{K}_{k}^{T} .
\end{aligned}
$$

(6) Determine whether resampling is required, continuously update the particle state until the iteration termination condition is satisfied, calculate the normalized weights for the particles, and update the particles:

$$
\widehat{x}_{k}^{i}=q\left(\mathbf{x}_{k}^{i} \mid \mathbf{x}_{0: k \mid k-1}^{i}, z_{1: k}\right)=N\left(\bar{x}_{k}^{i}, \widehat{P}_{k}^{i}\right),
$$

where $N($.$) represents the Gaussian distribution.$

\section{EEMD Noise Reduction Method}

3.1. Conventional EMD. Incident signals may be noisy when transmitted in high-temperature superconducting cables, and the accuracy of fault location can be improved by using suitable noise reduction methods. Empirical mode decomposition is a widely used adaptive time-frequency signal processing method, which can decompose the trends in the original signal at different scales one by one to obtain a finite number of intrinsic mode functions (IMFs), which contain local features at different time scales. The decomposition process is as follows:

(1) Use the method of cubic spline interpolation to find out all the maximal and minimal values of the signal sequence $y(t)$ and form the upper and lower envelopes $y_{\max }(t)$ and $y_{\min }(t)$. Calculate the mean value of the envelope avg $(t)$ and the deviation de $(t)$ of the mean and signal sequence.

$$
\begin{aligned}
\operatorname{avg}(t) & =\left[\frac{y_{\max }(t)+y_{\min }(t)}{2}\right], \\
\operatorname{de}(t) & =y(t)-\operatorname{avg}(t) .
\end{aligned}
$$


(2) Determine whether the deviation de $(t)$ meets the conditions of IMF: (1) the mean value of the envelope is always $0 ;(2)$ the difference between the number of poles and the number of zeros is not greater than 1 . If satisfied, the first IMF is recorded as $\zeta_{1}(t)=\operatorname{de}(t)$, and the difference between the IMF and the original signal can be obtained by $s u_{1}(t)=y(t)-\zeta_{1}(t)$; if not, repeat several times until the conditions are met.

(3) $s u_{1}(t)$ is taken as the new signal to be decomposed and keep repeating the above process until the remaining signal is a monotonic function.

$$
y(t)=\operatorname{su}_{n}(t)+\sum_{i=1}^{n} \zeta_{i}(t) .
$$

3.2. EEMD. The EMD has the shortage of mode mixing, which limits the noise reduction effect. To overcome this problem, EEMD introduces Gaussian white noise and achieves a better improvement effect. The decomposition steps are as follows:

(1) Add a zero-mean Gaussian white noise sequence to the original signal $y(t)$ to form a new sequence $y^{\prime}(t)$.

(2) Perform EMD on the new sequence $y^{\prime}(t)$ to obtain several IMFs.

(3) Repeat steps (1) and (2) several times, with different white noise added each time.

(4) Derive the mean value of the IMF obtained several times as the IMF of the EEMD.

\section{High-Temperature Superconducting Cable Fault Location Method Based on Improved Time-Frequency Domain Reflection Method and EEMD Noise Reduction}

In this paper, we propose a fault location method for hightemperature superconducting cables. Firstly, incident signals are sent to the cables, and the folded reflection signals generated by the incident signals at the impedance discontinuities can be obtained by the digital oscilloscope. Secondly, the time-frequency domain reflection method is applied to locate cable faults. The affine transformation is introduced to solve the cross-term interference problem of the time-frequency domain reflection method. The particle swarm algorithm is used to improve the accuracy of the stagger angle. Additionally, the unscented particle filter algorithm is employed to achieve cross-term separation and EEMD is introduced to denoise the signal to make the localization results more accurate. The steps of the method in this paper are as follows, and the flowchart is shown in Figure 2.

(1) Set up the simulation environment and model of high-temperature superconducting cable and set the appropriate parameters.

(2) Apply the TFDR method to send incident signal to the cable and use the digital oscilloscope to pick up

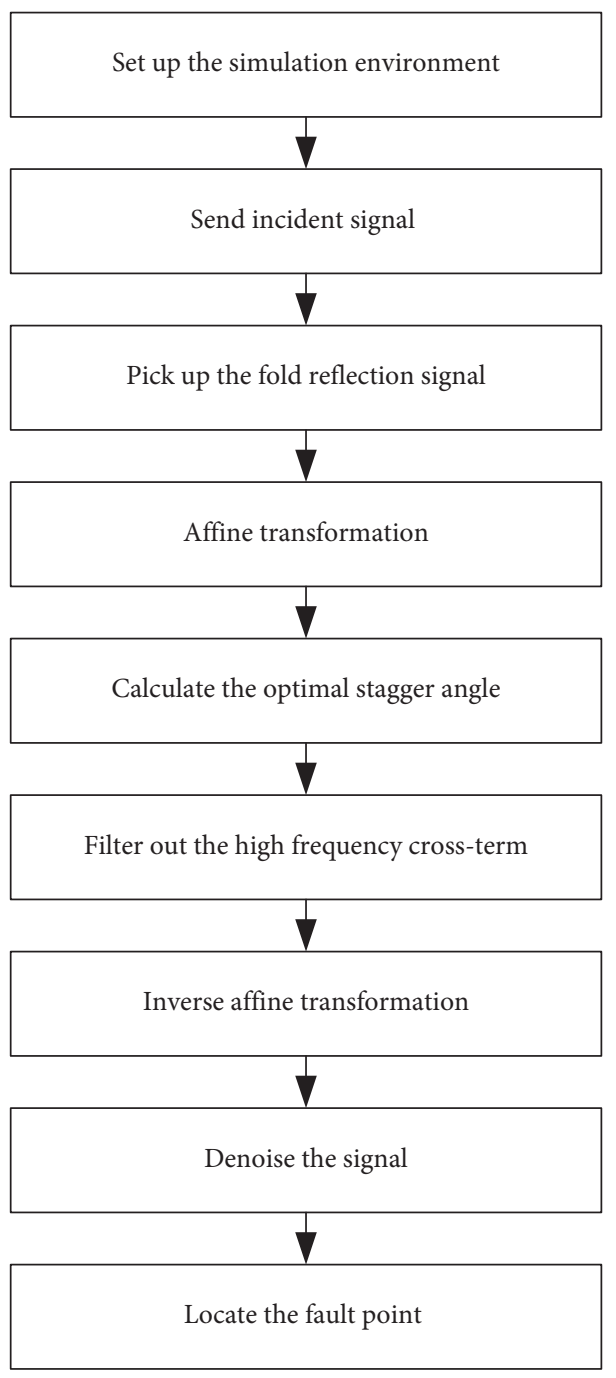

FIgURe 2: Algorithm flowchart.

the fold reflection signal generated by the incident signal at the impedance discontinuity point.

(3) The affine transformation is conducted to solve the cross-term interference problem.

(4) Particle swarm algorithm is used to improve the accuracy of the stagger angle.

(5) The unscented particle filter algorithm is employed to filter out the high-frequency cross term.

(6) The filtered transformed signal is inverse affine transformed back to the original coordinate system.

(7) Denoise the signal by EEMD.

(8) Locate the fault point based on the denoised folded reflection signal.

\section{Case Study}

5.1. High-Temperature Superconducting Cable Simulation Construction. In this paper, two software programs, PSCAD and MATLAB, are used for joint simulation. Since PSCAD does not have a model of high-temperature superconducting 
cable at the moment, it needs to be built manually. This study borrows the simulation construction method from the literature $[30,31]$ and uses the non-linear resistance instead of HTS in PSCAD, and the resistance value is calculated by writing the cable model in MATLAB, and the calculation block diagram is displayed in Figure 3.

As can be seen from Figure 3, during the PSCAD simulation, the HTS node currents obtained within each step are passed to the MATLAB program through the interface, and the cable resistance $R$ and cable temperature $T$ are calculated based on the cable model and then returned to PSCAD through the interface for display and completion of one step of simulation, and so on and so forth.

During actual operation, insulation faults and shortcircuit faults may occur in high-temperature superconducting cables. In this paper, the HTS model is established by PSCAD and MATLAB to simulate a $50 \mathrm{~m}$ long high-temperature superconducting cable with a three-phase short-circuit fault at $20 \mathrm{~m}$ from the head end. Since the high-temperature superconducting cable normally operates in a liquid nitrogen cryogenic environment, this paper assumes that the cable temperature during steady-state operation of the high-temperature superconducting cable is $80 \mathrm{~K}$.

5.2. Simulation Results. In order to verify the accuracy and validity of the method in this paper, the following six models are selected for comparison with the proposed method in this paper.

Case 1: the PWVD-TFDR model of Zheng [12].

Case 2: the GRNN-TFDR model of Rafinia and Moshtagh [13].

Case 3: the affine transformation-TFDR model of Liu et al. [17].

Case 4: the method proposed in this paper and that without EEMD noise reduction.

Case 5: the affine transformation-TFDR-particle swarm-Fourier filtering-EEMD noise reduction model is considered in this case.

Case 6: the affine transformation-TFDR-unscented particle filter-EEMD noise reduction is considered.

The localization polar spectrum of the above six control group models is shown in Figures 4-9, respectively, and the localization polar spectrum of the proposed method in this paper is depicted in Figure 10. For the convenience of observation, only each wave peak is labelled in the figure, and the remaining values are taken as 0 . The following conclusions can be drawn from the comparison analysis of the figures.

(1) The PWVD-TFDR cannot completely eliminate the cross-term interference, and there are more interference peaks.

(2) GRNN-TFDR has slightly less interference peaks than pseudo-Wigner distribution-TFDR, but the localization accuracy is not as good as the latter.

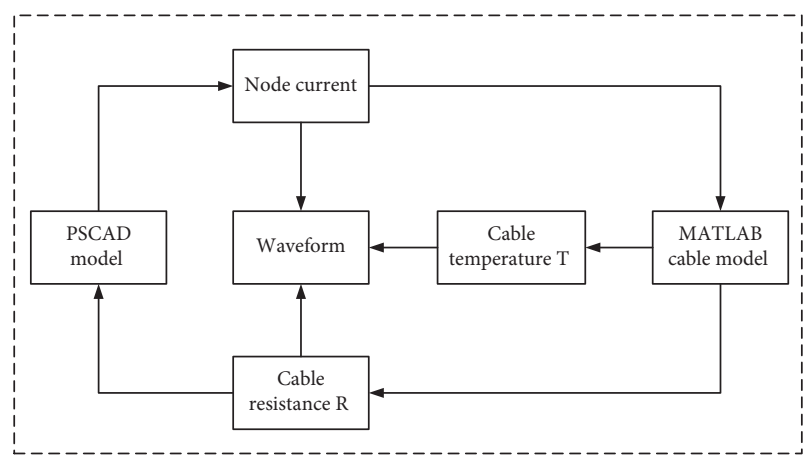

Figure 3: Calculation diagram of high-temperature superconducting cable model.

(3) The affine transformation-TFDR eliminates the interference peaks to a great extent, and there is only one small interference peak in the figure, and the localization accuracy is better than that of the pseudo-Wigner distribution-TFDR and GRNNTFDR, which verifies the advantage of the affine transformation idea in removing the cross-term interference.

(4) Compared with affine transformation-TFDR, affine transformation-TFDR-particle swarm-unscented particle filter further reduces the interference peaks and improves the localization accuracy, which verifies that particle swarm and unscented particle filter are beneficial to the accuracy improvement of the algorithm.

(5) Compared with the affine transformation-TFDR, the affine transformation-TFDR-particle swarm-Fourier filter-EEMD noise reduction further reduces the interference peaks and improves the localization accuracy, which verifies that the processing of particle swarm search for the stagger angle and EEMD noise reduction can improve the algorithm accuracy.

(6) Compared with the affine transformation-TFDR, the affine transformation-TFDR-unscented particle filter-EEMD noise reduction further reduces the interference peaks and improves the localization accuracy, and it is verified that the unscented particle filter and EEMD noise reduction are beneficial to the algorithm accuracy improvement.

The proposed method of this paper basically eliminates all the interference peaks and performs better compared with the 4 th, $5^{\text {th }}$, and 6 th control groups, which verifies that the three measures introduced in this paper, namely, particle swarm search for stagger angle, unscented particle filter for high-frequency cross terms, and EEMD noise reduction, are effective in improving the fault location accuracy.

The comparison of the positioning accuracy of each algorithm is shown in Table 1 . As can be seen from Table 1 , the algorithm of this paper outperforms the six control group algorithms, and the relative error is only $0.05 \%$, which is the smallest relative error of all algorithms, verifying the accuracy and effectiveness of the algorithm of this paper. 


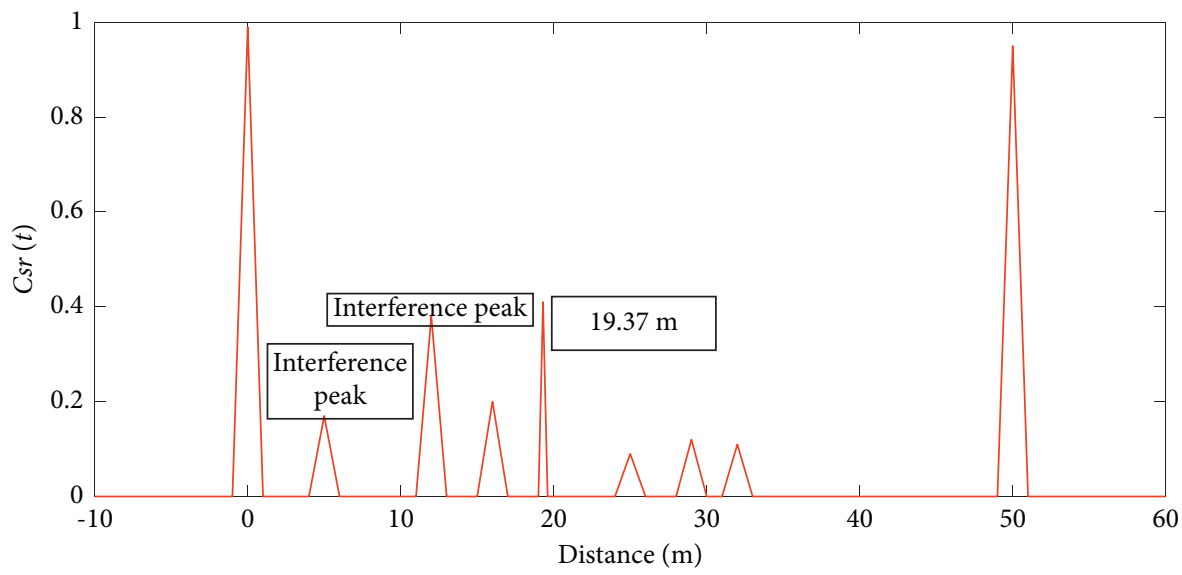

Figure 4: Positioning result of the PWVD-TFDR model.

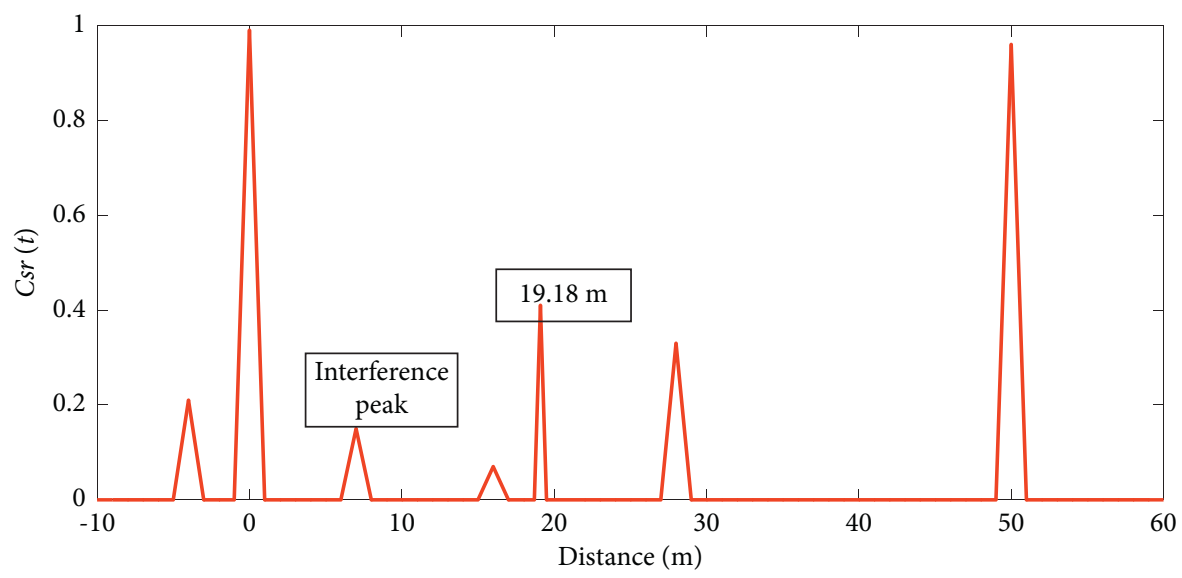

FIgure 5: Positioning result of the GRNN-TFDR model.

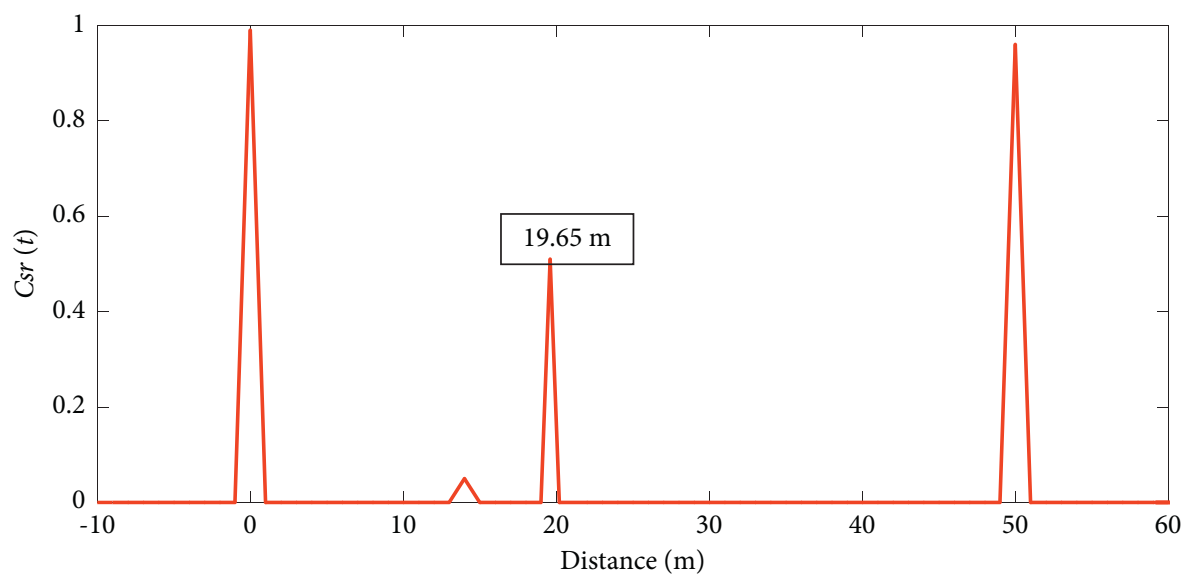

Figure 6: Positioning result of the affine transformation-TFDR model. 


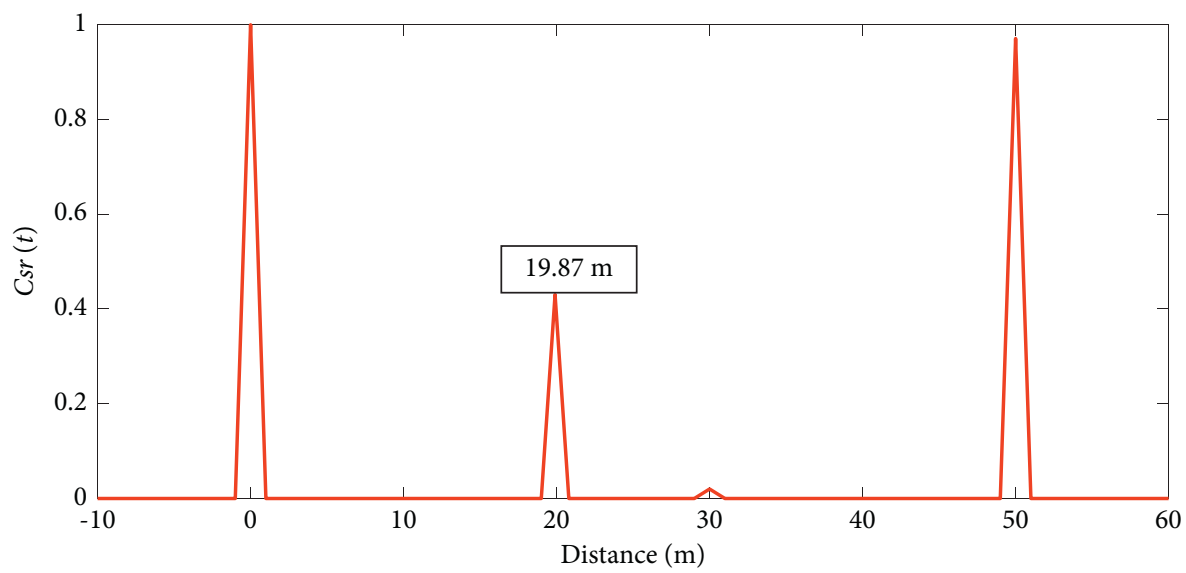

Figure 7: Positioning result of the affine transformation-TFDR-particle swarm-unscented particle filter model.

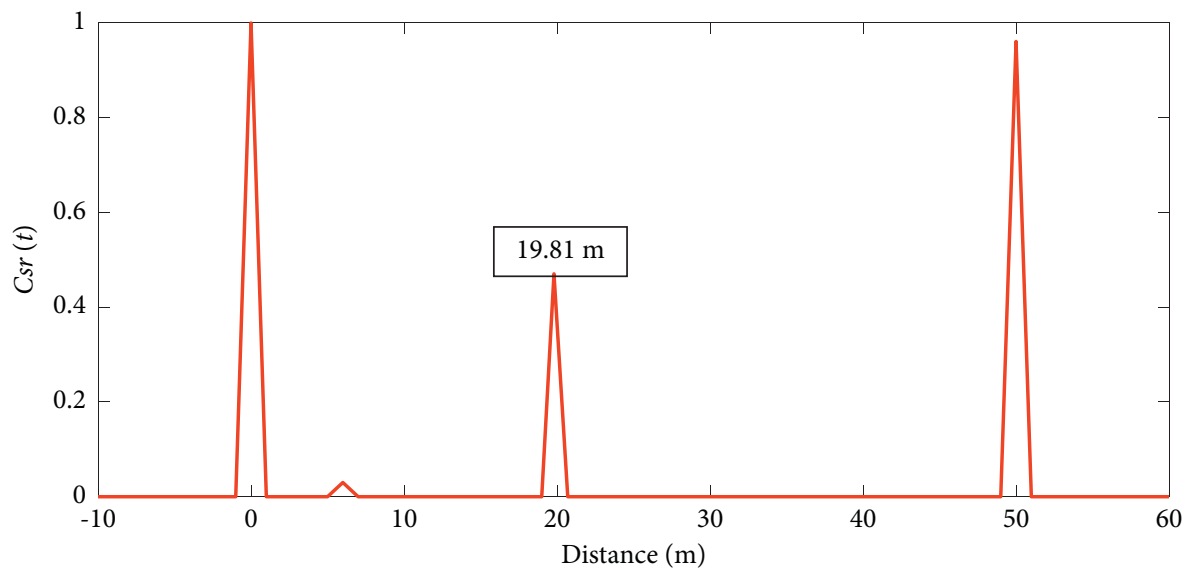

FIGURE 8: Positioning result of the affine transform-TFDR-particle swarm-Fourier filter-EEMD noise reduction model.

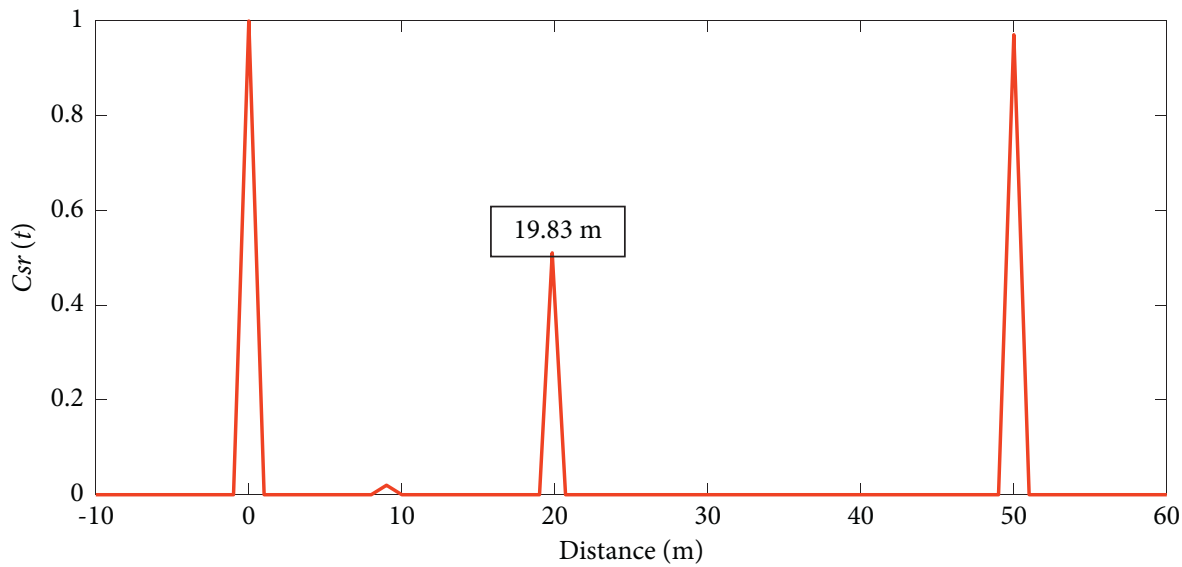

FIGURE 9: Positioning result of the affine transformation-TFDR-unscented particle filter-EEMD noise reduction model. 


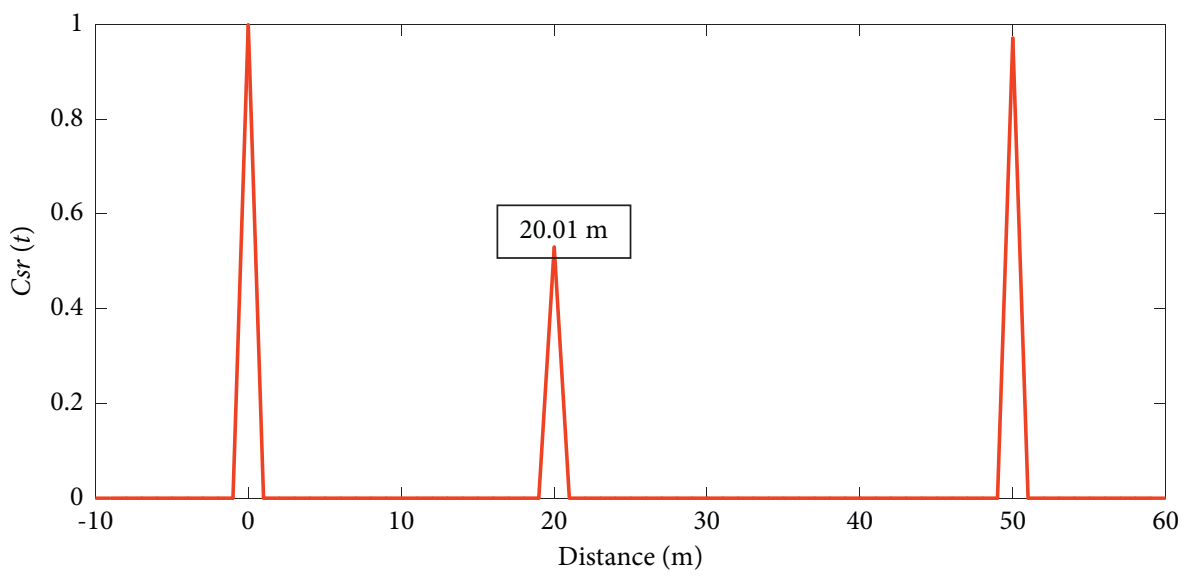

FIGURE 10: Positioning result of the proposed method.

TABLE 1: Comparison of positioning accuracy of various algorithms.

\begin{tabular}{lccc}
\hline Cases & Fault point $(\mathrm{m})$ & Absolute error $(\mathrm{m})$ & Relative error $(\%)$ \\
\hline Case 1 & 19.37 & 0.63 & 3.15 \\
Case 2 & 19.18 & 0.82 & 4.10 \\
Case 3 & 19.65 & 0.35 & 0.13 \\
Case 4 & 19.87 & 0.19 & 0.65 \\
Case 5 & 19.81 & 0.17 & 0.95 \\
Case 6 & 19.83 & 0.01 & 0.85 \\
The proposed method in this paper & 20.01 & & 0.05 \\
\hline
\end{tabular}

\section{Conclusions}

In this paper, a fault location method for high-temperature superconducting cables is proposed, which can improve the fault location accuracy by effectively solving the problems of self-term resolution reduction and incomplete cross-term removal that may be caused when removing cross-term interference.

This paper combines PSCAD and MATLAB software programs to build a high-temperature superconducting cable model, adopts the time-frequency domain reflection method to locate cable faults, introduces the affine transformation, applies particle swarm algorithm to improve the accuracy of the affine transformation stagger angle, realizes the cross-term separation according to the unscented particle filter algorithm, and finally performs EEMD denoising on the signal. The simulation results show that this method has good fault location accuracy for high-temperature superconducting cables, which has certain reference significance for engineering practice.

\section{Data Availability}

The data used to support the findings of this study are included within the article.

\section{Conflicts of Interest}

The authors declare that they have no conflicts of interest.

\section{Acknowledgments}

This study was supported by the Tongli High-Temperature Superconducting DC Cable Demonstration Project of Jiangsu Suzhou.

\section{References}

[1] M. Buhari, V. Levi, and S. K. E. Awadallah, "Modelling of ageing distribution cable for replacement planning," IEEE Transactions on Power Systems, vol. 31, no. 5, pp. 3996-4004, 2015.

[2] R. Bayindir, I. Colak, G. Fulli, and K. Demirtas, "Smart grid technologies and applications," Renewable and Sustainable Energy Reviews, vol. 66, pp. 499-516, 2016.

[3] C. Liu, Y. Zhang, and J. Sun, "Stacked bidirectional LSTM RNN to evaluate the remaining useful life of supercapacitor," International Journal of Energy Research, pp. 1-10, 2021.

[4] C. Lee, H. Son, and Y. Won, "Progress of the first commercial project of high-temperature superconducting cables by KEPCO in Korea," Superconductor Science and Technology, vol. 33, no. 4, 2020.

[5] G. Xia, Y. Huang, F. Li et al., "A thermally flexible and multisite tactile sensor for remote $3 \mathrm{D}$ dynamic sensing imaging," Frontiers of Chemical Science and Engineering, vol. 14, no. 6, pp. 1039-1051, 2020.

[6] J. H. Choi, H. Park, and R. Baldick, "Transmission investment and expansion planning for systems with high-temperature superconducting cables," IEEE Transactions on Applied Superconductivity, vol. 29, no. 8, pp. 1-9, 2019.

[7] O. Rahman, K. M. Muttaqi, and D. Sutanto, "High temperature superconducting devices and renewable energy 
resources in future power grids: a case study," IEEE Transactions on Applied Superconductivity, vol. 29, no. 2, pp. 1-4, 2019.

[8] J. X. Jin, Y. Ying Xin, Q. L. Wang et al., "Enabling hightemperature superconducting technologies toward practical applications," IEEE Transactions on Applied Superconductivity, vol. 24, no. 5, pp. 1-12, 2014.

[9] W. Yuan, S. Venuturumilli, Z. Zhang, Y. Mavrocostanti, and M. Zhang, "Economic feasibility study of using high-temperature superconducting cables in U.K.'s electrical distribution networks," IEEE Transactions on Applied Superconductivity, vol. 28, no. 4, pp. 1-5, 2018.

[10] Z. Feng, C. H. I. Zhen, and F. U. Sheng-Qun, "Design of the pulse source of cable fault location based on pulse reflection method," Journal of Harbin University of Science \& Technology, vol. 19, no. 3, 2014.

[11] S. Y. Tao, Y. Feng, and T. C. Zhang, "High-voltage switch cabinet partial discharge on-line monitoring device based on pulse current method," Power System Protection and Control, vol. 47, no. 9, pp. 145-149, 2019.

[12] G. M. Zheng, "A reflectometer for cable fault location with multiple pulse reflection method," Sensors \& Transducers, vol. 183, no. 12, 2014.

[13] A. Rafinia and J. Moshtagh, "A new approach to fault location in three-phase underground distribution system using combination of wavelet analysis with ANN and FLS," International Journal of Electrical Power \& Energy Systems, vol. 55, pp. 261-274, 2014.

[14] J. P. Huang, Z. Zhang, and W. Wang, "A local quench detection and protection method for a superconducting cable based on distributed optical fiber temperature measurement technology," Power System Protection and Control, vol. 48, no. 14 , pp. 76-84, 2020.

[15] Y. Y. Wang, Z. F. Yao, and W. Xie, "Research on fault location of high temperature superconducting cable based on timefrequency domain reflectometry," Proceedings of the CSEE, vol. 41, no. 5, pp. 1540-1546, 2021.

[16] G.-Y. Kwon, C.-K. Lee, and Y.-J. Shin, "Diagnosis of shielded cable faults via regression-based reflectometry," IEEE Transactions on Industrial Electronics, vol. 66, no. 3, pp. 2122-2131, 2019.

[17] C. Liu, Q. Li, and K. Wang, "State-of-charge estimation and remaining useful life prediction of supercapacitors," Renewable and Sustainable Energy Reviews, vol. 150, no. 2, p. 111408, 2021.

[18] Y. H. Lee, S. S. Bang, and G. Y. Kwon, "Analysis of wave propagation of HTS cables for compensation of thermal loss on connectors," IEEE Transactions on Applied Superconductivity, vol. 27, no. 4, p. 4801105, 2017.

[19] G.-Y. Kwon, C.-K. Lee, G. S. Lee et al., "Offline fault localization technique on HVDC submarine cable via time-frequency domain reflectometry," IEEE Transactions on Power Delivery, vol. 32, no. 3, pp. 1626-1635, 2017.

[20] K. Wang, C. L. Liu, and J. R. Sun, "State of charge estimation of composite energy storage systems with supercapacitors and lithium batteries," Complexity, vol. 2021, Article ID 8816250, 15 pages, 2021.

[21] J. Chang, L. Zhu, H. Li, F. Xu, B. Liu, and Z. Yang, "Noise reduction in Lidar signal using correlation-based EMD combined with soft thresholding and roughness penalty," Optics Communications, vol. 407, pp. 290-295, 2018.

[22] W.-C. Wang, K.-W. Chau, D.-M. Xu, and X.-Y. Chen, "Improving forecasting accuracy of annual runoff time series using ARIMA based on EEMD decomposition," Water Resources Management, vol. 29, no. 8, pp. 2655-2675, 2015.
[23] X. Feng, Q. Li, and K. Wang, "Waste plastic triboelectric nanogenerators using recycled plastic bags for power generation," ACS Applied Materials \& Interfaces, vol. 13, no. 1, pp. 400-410, 2020.

[24] B. Zhang, Z. Zou, and S. J. Chen, "Fabric image registration based on affine transform and Levenberg-Marquardrt algorithm," Acta Optica Sinica, vol. 37, no. 1, 2017.

[25] K. Wang, W. Wang, L. Wang, and L. Li, "An improved SOC control strategy for electric vehicle hybrid energy storage systems," Energies, vol. 13, no. 20, p. 5297, 2020.

[26] R. A. Ibrahim, A. A. Ewees, D. Oliva, M. Abd Elaziz, and S. Lu, "Improved salp swarm algorithm based on particle swarm optimization for feature selection," Journal of Ambient Intelligence and Humanized Computing, vol. 10, no. 8, pp. 3155-3169, 2019.

[27] S. Almahdi and S. Y. Yang, “A constrained portfolio trading system using particle swarm algorithm and recurrent reinforcement learning," Expert Systems with Applications, vol. 130, pp. 145-156, 2019.

[28] H. Zhang, Q. Miao, X. Zhang, and Z. Liu, "An improved unscented particle filter approach for lithium-ion battery remaining useful life prediction," Microelectronics Reliability, vol. 81, pp. 288-298, 2018.

[29] G. Vezzani, U. Pattacini, G. Battistelli, L. Chisci, and L. Natale, "Memory unscented particle filter for 6-DOF tactile localization," IEEE Transactions on Robotics, vol. 33, no. 5, pp. 1139-1155, 2017.

[30] X. Y. Chen, Experimental and Simulation on $110 \mathrm{kV}$ Cold Dielectric High Temperature Superconducting Cable, Beijing Jiaotong University, Beijing, China, 2015.

[31] Y. X. Li, The Study of HTS Model Cable Simulation Model Based on PSCAD, School of Electrical and Electronic Engineering, Beijing, China, 2014. 